\title{
Acute Torsion of the Gallbladder
}

\author{
Haithem Zaafouri*, Skander Mrad, Noomen Haoues, Rabii Noomene and Abderraouf Cherif \\ Department of general surgery, Habib Thameur hospital, 8 Ali Ben Ayed Street's, Monftlleury 1008, Tunis, Tunisia
}

Received: June 05, 2014; Accepted: July 10, 2014; Published: July 22, 2014

*Corresponding author: Haithem Zaafouri, Associate professor, Habib Thameur hospital, Tunisia, Tel: +21697348839; E-mail: zaafouri.haithem@ hotmail.fr

\section{Introduction}

Gallbladder volvulus is a rare cause of a traumatic, acute abdomen which is often very difficult to diagnose pre-operatively. Precise etiology of gallbladder torsion remains unknown, but it is believed that it occur in anatomically predisposed patients. Preoperative diagnosis of gallbladder torsion remains difficult and most cases are found as a surprise at surgery.

This case demonstrated that gallbladder torsion is another possible mechanism for acute gangrenous cholecystitis.

\section{Case Presentation}

An 89 year old man presented to the emergency department with a two days history of acute onset abdominal pain in the upper right quadrant, with fever, vomiting and malaise. His medical history included peptic ulcer disease and tobacco abuse. There was no significant relevant past surgical history. He was dehydrated at presentation with the following vital signs: HR$86 \mathrm{~b} / \mathrm{min}, \mathrm{BP}-100 / 50 \mathrm{mmHg}, \mathrm{T}-38.5^{\circ} \mathrm{C}$. Focused abdominal examination demonstrated tenderness to palpation in the right upper quadrant, and a positive Murphy's sign. Laboratory blood tests revealed a leukocytosis of $22.7 \times 103 / \mathrm{L}$, C-reactive protein of $48 \mathrm{mg} / \mathrm{L}$ and normal kidney and liver function tests. Abdominal ultrasonography (Figure 1) and computed tomography (CT) scan (Figure 2) showed a clearly enlarged gallbladder with a thickened wall of $7 \mathrm{~mm}$, with fluid sub-hepatically. They demonstrated no gallstone. Free air within the gallbladder wall was not seen. He was admitted to our hospital with the diagnosis of acute cholecystitis. After resuscitation emergency laparotomy through a midline incision was performed. On entering the abdominal cavity, a gangrenous distended gallbladder with omentum adhesed to it circumferentially was immediately noted (Figure 3). It was rotated more than 360 degrees anticlockwise around its mesentery. The gallbladder torsion was reduced and a cholecystectomy was then performed in the standard fashion, with placement of a drain in the gallbladder fossa. No gallbladder stones were found in the specimen. Histology revealed transmural necrosis consistent with volvulus. His post-operative course was unremarkable and he was discharged on post-operative day 3 .

\section{Discussion}

Gallbladder volvulus occurs predominantly in older people

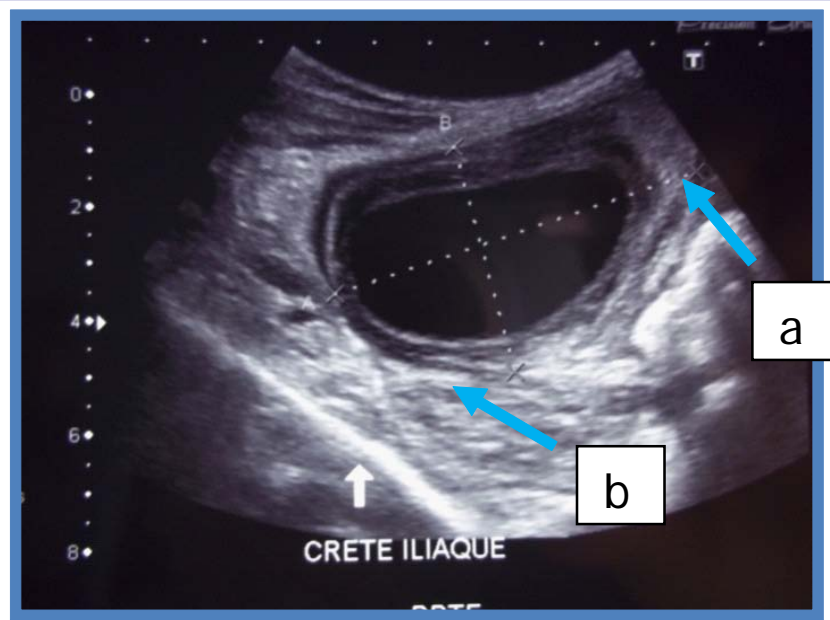

Figure 1: Transverse ultrasonogram of the gallbladder shows a markedly thickened gallbladder wall (a) and fluid collection (b).

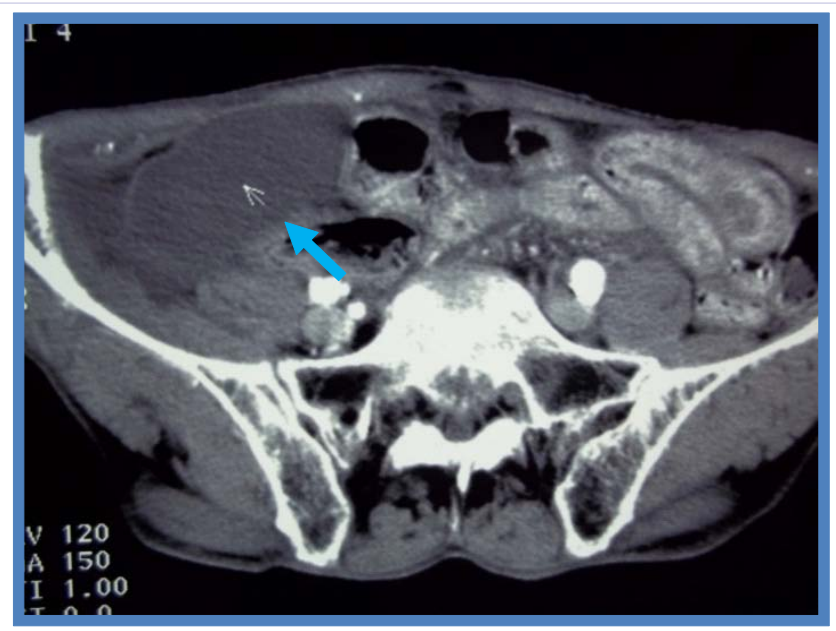

Figure 2: Abdominal CT scan demonstrates a markedly distended gallbladder.

with a female to male sex ratio of 3:1 [1]. Since its first description in 1898 by Wendel [2], there have been over 500 documented cases in the literature.

The etiology is thought that a long gallbladder mesentery with 


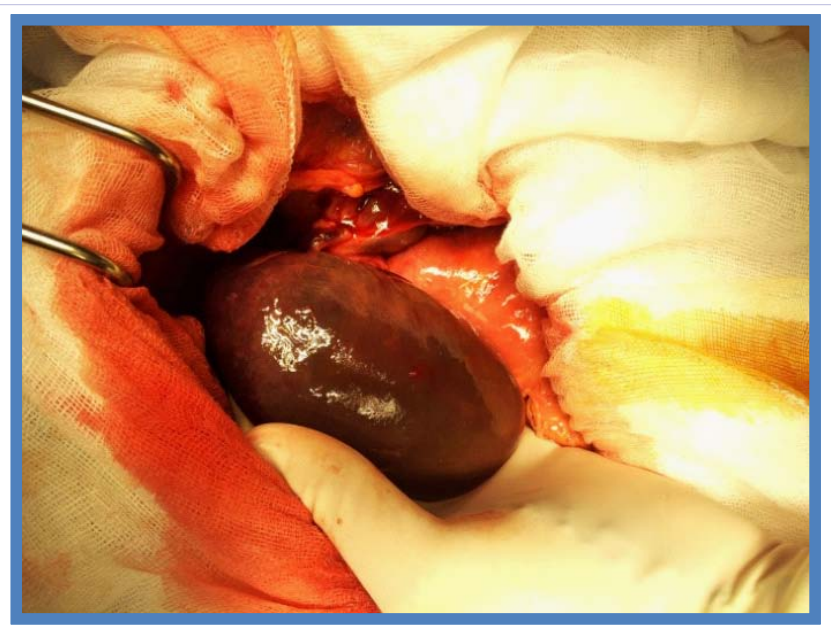

Figure 3: Torsion of the gall bladder with gangrene.

minimal or no attachment to the liver facilitates the longitudinal axis of the gallbladder to twist on its vascular root resulting in ischemia, necrosis and perforation [3,4]. Boonstra [5] suggested that torsion happens only in the absence of hepatic attachments and fixation to the liver. The disease carries a significant morbidity and mortality due to the risk of necrosis and perforation of the gallbladder. The reported mortality in the literature is up to $6 \%$ [6].

Gallbladder volvulus is frequently misdiagnosed as acute acalculous cholecystitis, as no single clinical, serologic, or radiographic finding is pathognomonic. To make the diagnosis of gallbladder torsion is difficult, with less than $10 \%$ of cases reported pre-operatively in the literature [7].

The particularity of our case is that the patient is male and he had the triad (Elderly, Right upper quadrant Pain, Pulsetemperature discrepancy) of triads described by Lau [8].

Abdominal computer tomography (CT) with iodinated contrast injection shows the gallbladder to be horizontal and located outside of the gallbladder bed together with torsion of the cystic pedicle and a "whirl sign". It is worth noting that 3-dimensional reconstructed CT may be useful in preoperative diagnosis of gallbladder torsion [9].

Treatment is surgical with urgent cholecystectomy because of the risk of gallbladder perforation. Initial release of the torsion on the gallbladder can reduce the risk of damage to the biliary tract.
Prognosis is excellent if expeditious cholecystectomy is performed. If treatment is delayed, infarction and perforation resulting in bilious peritonitis increase the mortality rate to approximately $5 \%$.

\section{Conclusion}

Gallbladder volvulus is a rare cause of acute cholecystitis, which is occasionally difficult to diagnose pre-operatively. Having a high index of suspicion for other causes of acute cholecystitis in the absence of simple gall stone obstruction, or when investigation results do not correlate with the clinical presentation, gallbladder torsion should still be considered even if it is an extremely rare entity. Gallbladder torsion mandates emergent cholecystectomy, to avoid visceral perforation, bilious peritonitis, and hemodynamic instability.

\section{References}

1. Alkhalili E, Bencsath K. Gallbladder torsion with acute cholecystitis and gross necrosis. BMJ Case Rep. 2014. doi: 10.1136/bcr-2014204917.

2. Pottorf BJ, Alfaro L, Hollis HW. A Clinician's Guide to the Diagnosis and Management of Gallbladder Volvulus. Perm J. 2013; 17(2):80-3. doi: 10.7812/TPP/12-118.

3. Chalret du Rieu M, Carrere N. Volvulus de la vesicule biliaire. Journal de Chirurgie Viscerale. 2012; 149(2):172-3

4. Gabizon S, Bradshaw K, Jeyarajan E, Alzubaidy R, and Liew V. Gallbladder Torsion: A Diagnostic Challenge. Case Rep Surg. 2014. doi: $10.1155 / 2014 / 902814$.

5. Boonstra EA, van Etten B, Prins TR, Sieders E, van Leeuwen BL. Torsion of the Gallbladder. J Gastrointest Surg. 2012;16(4):882-4. doi: 10.1007/s11605-011-1712-6.

6. Reilly DJ, Kalogeropoulos G, Thiruchelvam D. Torsion of the Gallbladder: a systematic review. HPB (Oxford). 2012;14(10):669-72. doi: 10.1111/j.1477-2574.2012.00513.x.

7. Boer J, Boerma D, and De Vries Reilingh TS. A gallbladder torsion presenting as acute cholecystitis in an elderly woman: a case report. J Med Case Rep. 2011; 5(1):588. doi: 10.1186/1752-1947-5-588.

8. Lau WY, Fan ST, Wong SH. Acute torsion of the gall bladder in the aged: a re-emphasis on clinical diagnosis. Aust N Z J Surg. 1982; 52(5):492-4.

9. Yokoi T, Miyata K, Yuasa N, Takeuchi E, Goto Y, Miyake H, et al. Twisted cystic artery disclosed by 3-dimensional computed tomography angiography for torsion of the gallbladder. Am J Surg. 2011; 201(5):33-4. doi: 10.1016/j.amjsurg.2010.04.026. 